01

\title{
О внутридопплеровской спектроскопии в тонких газовых ячейках при возбуждении атомов широкополосным импульсным излучением
}

\author{
(C) А.Ч. Измайлов \\ Институт фризики НАН Азербайджана, \\ AZ-1143 Баку, Азербайджан \\ e-mail: azizm57@rambler.ru
}

Поступила в редакцию 01.09.2019 г.

В окончательной редакции 20.11.2019 г.

Принята к публикации 22.11.2019 г.

\begin{abstract}
Предложен метод внутридопплеровской спектроскопии, основанный на особенностях сужения с течением времени скоростного распределения оптически возбужденных атомов (молекул) разреженной газовой среды в тонкой ячейке после воздействия достаточно короткого импульса широкополосного излучения. Регистрация узких внутридопплеровских резонансов на центральных частотах квантовых переходов из атомного состояния, возбужденного таким импульсом накачки, осуществляется последующими пробными монохроматическими световыми импульсами. Оптимальные условия для реализации данного метода спектроскопии имеют место, когда время радиационного распада оптически возбужденного атомного уровня превосходит характерное время пролета атомов между стенками рассматриваемой тонкой ячейки, внутренняя толщина которой во много раз меньше ее поперечных размеров.
\end{abstract}

Ключевые слова: внутридопплеровская спектроскопия, тонкая газовая ячейка, широкополосная импульсная накачка, долгоживущие квантовые уровни.

DOI: $10.21883 /$ OS.2020.03.49054.264-19

\section{Введение}

Для спектроскопии сверхвысокого разрешения атомов и молекул очень важна разработка эффективных методов, которые позволяют анализировать структуру спектральных линий, скрытую допплеровским уширением [1]. Так, в обзоре [2] описаны достижения и возможности внутридопплеровской спектроскопии, основанной на особенностях пролетной релаксации и оптической накачке атомов в тонких газовых ячейках, внутренняя толщина которых во много раз меньше их характерных поперечных размеров. К настоящему времени достаточно хорошо разработаны и апробированы методы спектроскопии в таких ячейках при стационарной оптической накачке газовой среды. Вместе с тем японские ученые реализовали на эксперименте метод внутридопплеровской спектроскопии в тонкой ячейке (с внутренней толщиной $l=1 \mathrm{~mm}$ и диаметром $d=34 \mathrm{~mm}$ ) с разреженными парами рубидия на основе нестационарных фотопроцессов [3]. В цитированной работе осуществлялась начальная накачка импульсом монохроматического излучения с частотой около центров переходов линии $D_{1}$ атомов ${ }^{85} \mathrm{Rb}$. Через определенные промежутки времени после воздействия этого импульса накачки пары рубидия в ячейке зондировались сравнительно слабыми световыми импульсами такой же частоты и направления (от того же диодного лазера). Таким способом удается выявить вклад сравнительно медленных оптически накачанных атомов ${ }^{85} \mathrm{Rb}$ в основном квантовом состоянии, которые не успевают столкнуться со стенками ячейки в течение некоторого времени. Поэтому посредством контролируемого изменения времен задержки между накачивающим и пробными световыми импульсами в работе [3] были зарегистрированы узкие внутридопплеровские резонансы поглощения на центрах оптических переходов линии $D_{1}$ атомов ${ }^{85} \mathrm{Rb}$ при сканировании лазерной частоты. В последующей работе [4] проведено теоретическое исследование селективных внутридопплеровских фотопроцессов в тонких газовых ячейках при подобной монохроматической импульсной накачке.

Вместе с тем эффективная селекция сравнительно медленных атомов на определенных квантовых уровнях в тонких газовых ячейках возможна также при их стационарной накачке широкополосным излучением. Тогда посредством дополнительного пробного монохроматического пучка могут быть зарегистрированы узкие внутридопплеровские резонансы на центрах оптических переходов не только из основного квантового терма, но также из возбужденных атомных уровней [2,5]. $\mathrm{B}$ настоящей работе показано, что для спектроскопии сверхвысокого разрешения в тонких газовых ячейках эффективно использование также импульсной широкополосной накачки.

\section{Основные соотношения}

Рассмотрим разреженную газовую среду в ячейке, сделанной из прозрачного материала в форме прямоугольного параллелепипеда (рис. 1). Внутренняя толщина $l$ этой ячейки (вдоль оси $z$ ) во много раз меньше ее характерного поперечного размера $d$ (вдоль осей $x$ и $y$ ). 


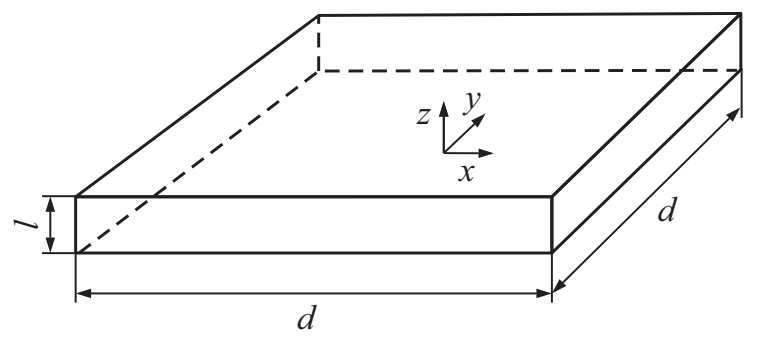

Рис. 1. Газовая ячейка в форме прямоугольного параллелепипеда с малой внутренней толщиной: $l \ll d$.

Газовая среда в ячейке полагается настолько разреженной, что взаимодействием между атомами (молекулами) газа в ней можно пренебречь. Данная среда в течение интервала времени $-T \leq t \leq 0$ облучается достаточно коротким импульсом широкополосной накачки, которая переводит атомы из основного уровня $a$ в возбужденное состояние $b$. После воздействия такого импульса населенность возбужденного уровня $b$ уменьшается со временем не только вследствие его радиационного распада (с характерным временем $\tau_{b}$ ), но также в результате столкновений атомов (молекул) со стенками ячейки. Таким образом, через некоторое время $t>0$ в рассматриваемой ячейке (рис. 1) могут остаться только такие возбужденные атомы, чьи проекции скоростей $v_{x}$, $v_{y}$ и $v_{z}$ на оси $x, y$ и $z$ удовлетворяют следующим условиям:

$$
\left|v_{x}\right| t \leq d,\left|v_{y}\right| t \leq d,\left|v_{z}\right| t \leq l
$$

Далее будем полагать длительность $T$ импульса настолько короткой, что выполняются соотношения $T \ll \tau_{b}$ и $T \ll l / u$, где $u$ - наиболее вероятная скорость атомов в газе. Тогда в течение этого импульса можно пренебречь как радиационной релаксацией возбужденных частиц, так и относительным количеством данных частиц, которые, согласно (1), успевают столкнуться со стенками ячейки. При рассматриваемых условиях имеет место следующая система уравнений для относительных населенностей $\rho_{a}$ и $\rho_{b}$ уровней $a$ и $b$ в течение столь короткого импульса широкополосной накачки на переходе $a \rightarrow b[1]:$

$$
\begin{gathered}
\rho_{a}+\rho_{b}=1, \\
\frac{d \rho_{a}}{d t}=\theta(t)\left(\rho_{b}-\rho_{a}\right), \\
\frac{d \rho_{b}}{d t}=\theta(t)\left(\rho_{a}-\rho_{b}\right),
\end{gathered}
$$

где $\theta(t)$ - скорость оптической накачки, которая полагается однородной по всему объему ячейки (рис. 1). До облучения газовой среды в равновесном состоянии относительные населенности уровней $a$ и $b$ атомов равны соответственно 1 и 0 . Тогда из системы уравнений (2) получаем следующее выражение для населенности возбужденного уровня $b$ к моменту $t=0$ окончания импульса накачки:

$$
\rho_{b}^{(0)}=0.5\left\{1-\exp \left[-2 \int_{0}^{T} \theta\left(t_{1}\right) d t_{1}\right]\right\} .
$$

Изменение населенности $\rho_{b}$ данного уровня $b$ в течение последующего времени $t>0$ описывается следующим уравнением для атомов (молекул) со скоростью $\mathbf{v}$ и координатным вектором $\mathbf{r}[1]$ :

$$
\frac{\partial \rho_{b}}{\partial t}+\mathbf{v} \frac{\partial \rho_{b}}{\partial \mathbf{r}}+\frac{\rho_{b}}{\tau_{b}}=0
$$

Наряду с начальным условием $\rho_{b}(t=0)=\rho_{b}^{(a)}(3)$, уравнение (4) должно быть дополнено граничными условиями, которые зависят от особенностей столкновений атомов (молекул) со стенками ячейки. Как и в предыдущих теоретических исследованиях $[2,4,5]$ для тонких газовых ячеек, будем считать, что в результате таких столкновений устанавливается равновесное распределение как по скоростям атомов, так и по населенностям их квантовых уровней. Тогда возбужденные на уровень $b$ атомы релаксируют в основное квантовое состояние $a$ при любом их столкновении с внутренней поверхностью ячейки. При рассматриваемых условиях из уравнения (4) получаем следующее выражение для относительной населенности $\rho_{b}(\mathbf{r}, \mathbf{v}, t)$ возбужденных атомов в случае газовой ячейки (рис. 1), ограниченной шестью стенками с координатами $z= \pm 0.5 l, x= \pm 0.5 d$ и $y= \pm 0.5 d$ :

$$
\begin{aligned}
& \rho_{b}(\mathbf{r}, \mathbf{v}, t)=\rho_{b}^{(0)} \exp \left(-t / \tau_{b}\right) \eta(0.5 l-|z|) \\
& \quad \times \eta(0.5 d-|x|) \eta(0.5 d-|y|) \sum_{\alpha= \pm 1} \sum_{\beta= \pm 1} \sum_{\sigma= \pm 1}\left\{\eta\left(\alpha v_{x}\right)\right. \\
& \quad \times \eta\left(\beta v_{y}\right) \eta\left(\sigma v_{z}\right)\left[0.5 d+\alpha\left(x-v_{x} t\right)\right] \eta\left[0.5 d+\beta\left(y-v_{y} t\right)\right] \\
& \left.\quad \times \eta\left[0.5 l+\sigma\left(z-v_{z} t\right)\right]\right\}
\end{aligned}
$$

где $\eta(p)$ - ступенчатая функция $(\eta(p)=1$, если $p \geq 0$, и $\eta(p)=0$, если $p<0)$. Далее будем анализировать динамику изменения распределения $N_{b}\left(v_{z}, t\right)$ возбужденных атомов по проекции атомной скорости $v_{z}$ во всем объеме $V$ рассматриваемой ячейки:

$$
\begin{aligned}
N_{b}\left(v_{z}, t\right)= & n_{a} F\left(v_{z}\right) \int_{-\infty}^{+\infty}\left[\int \rho_{b}(\mathbf{r}, \mathbf{v}, t) d^{3} \mathbf{r}\right] \\
& \times F\left(v_{x}\right) F\left(v_{y}\right) d v_{x} d v_{y},
\end{aligned}
$$

где $n_{a}$ - равновесная плотность атомов в основном состоянии $a, F\left(v_{j}\right)$ - распределение Максвелла по проекции скорости $v_{j}$ :

$$
F\left(v_{j}\right)=\pi^{-0.5} u^{-1} \exp \left(-v_{j}^{2} / u^{2}\right), \quad(j=x, y, z)
$$


с наиболее вероятной скоростью $u$ атомов в газе. Подставляя выражение (5) в (6), получаем

$$
\begin{aligned}
N_{b}\left(v_{z}, t\right)= & N_{0} \rho_{b}^{(0)} F\left(v_{z}\right) \exp \left(-t / \tau_{b}\right) s(t) \\
& \times\left(1-\left|v_{z}\right| t l^{-1}\right) \eta\left(1-\left|v_{z}\right| t l^{-1}\right),
\end{aligned}
$$

где $N_{0}=n_{a} V-$ количество атомов во всем объеме $V=l d^{2}$ ячейки (рис. 1). Функция $s(t)$ в (8) имеет вид

$$
s(t)=4\left[\int_{0}^{d / t} F(v)\left(1-v t d^{-1}\right) d v\right]^{2}, \quad(t \geq 0)
$$

с зависимостью $F(v)(7)$ и описывает уменьшение количества возбужденных атомов со временем $t \geq 0$ вследствие их столкновений с боковыми стенками ячейки с координатами $x= \pm 0.5 d$ и $y= \pm 0.5 d$ (рис. 1). Эта функция (9) монотонно убывает, начиная со значения $s(0)=1$, причем $s(t) \approx \pi^{-1} d^{2} u^{-2} t^{-2}$, когда $t>2 d / u$.

\section{Обсуждение результатов}

На рис. 2 представлены распределения $N_{b}\left(v_{z}, t\right)(8)$ возбужденных атомов в тонкой ячейке (рис. 1) по их проекции скорости $v_{z}$ в различные моменты времени $t$ после воздействия импульса широкополосной накачки. Согласно (8), при $t \gg l / u$ характерная ширина $w(t)$ такого скоростного распределения имеет вид

$$
w(t)=2 l / t
$$

Уменьшение амплитуды $A(t)=N_{b}\left(v_{z}=0, t\right)$ данного распределения $N_{b}\left(v_{z}, t\right)(8)$ со временем $t$ описывается следующим выражением при $t>2 d / u$ :

$$
A(t) \approx \pi^{-1.5} N_{0} \rho_{b}^{(0)} \exp \left(-t / \tau_{b}\right) d^{2} u^{-3} t^{-2} .
$$

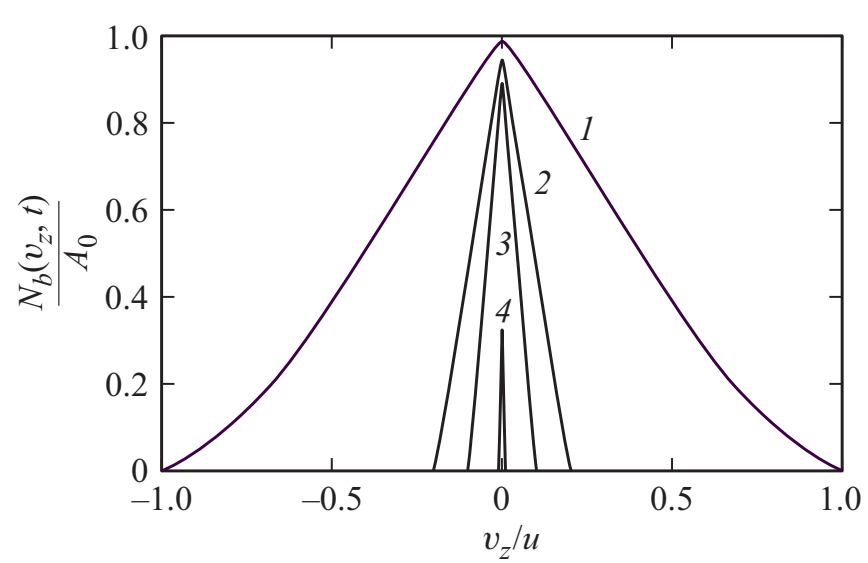

Рис. 2. Распределение $N_{b}\left(v_{z}, t\right)$ возбужденных на уровень $b$ атомов (в ед. $A_{0}=N_{b}\left(v_{z}=0, t=0\right)$ ) по проекции скорости $v_{z}$ в тонкой газовой ячейке при $d=10^{3} l$ и $\tau_{b}=10^{2} l / u$ для различных значений отношения $t /(l / u)=1(1), 5(2), 10$ (3) и 100 (4) после воздействия импульса широкополосной оптической накачки.
Формально ширина $w(t)(10)$ скоростного распределения возбужденных атомов может достигать сколь угодно малой величины с ростом времени $t$. Однако, согласно (11), амплитуда $A(t)$ этого распределения уменьшается гораздо быстрее, когда $t>\tau_{b}$ и $t>2 d / u$.

Очевидно, что наиболее эффективная скоростная селекция атомов (молекул) с малой проекцией их скорости $\left|v_{z}\right|$ рассмотренным способом возможна на сравнительно долгоживущих возбужденных квантовых уровнях. Такая ситуация имеет место, например, для метастабильных и ридберговских состояний атомов и молекул [6]. Тогда время радиационного распада $\tau_{b}>10^{-4}$ s подобных уровней будет превосходить время $d / u$ пролета атомов (молекул) с характерной скоростью $u \sim 10^{2} \mathrm{~m} / \mathrm{s}$ через тонкую ячейку (рис. 1) с поперечным размером $d \sim 1 \mathrm{~cm}$. Заметим, что через временной интервал, равный времени пролета $t \sim d / u$, ширина скоростного распределения $N_{b}\left(v_{z}, t\right)$ возбужденных атомов будет равна $w \sim(l / d) u \ll u(10)$. Например, для изготавливаемых к настоящему времени ультратонких газовых ячеек [7] с внутренней толщиной $l \sim 10 \mathrm{~nm}$ и поперечными размерами $d \sim 1 \mathrm{~cm}$ ширина $w \sim(l / d) u$ на 6 порядков меньше наиболее вероятной скорости $u$ атомов (молекул) газа. До такой же величины $w / u \ll 1$ уменьшится и допплеровское уширение регистрируемых спектральных линий на переходах из возбужденного уровня $b$.

Таким образом, через определенные промежутки времени после воздействия широкополосного импульса накачки в тонкой ячейке (рис. 1) остаются лишь возбужденные атомы с весьма малыми проекциями скорости $\left|v_{z}\right|$. Совокупность таких атомов в рассматриваемой ячейке фактически является компактным аналогом атомного пучка с эффективной расходимостью $w(t) / u \ll 1$, которая уменьшается со временем $t$ согласно формуле (10). Тогда подобно экспериментальной методике, разработанной в упомянутой работе [3], из возбужденного уровня $b$ можно осуществить спектроскопию сверхвысокого разрешения на квантовых переходах в другие уровни атомов (молекул) посредством контролируемых по времени и частоте импульсов пробного монохроматического излучения, распространяющихся вдоль оси $z$ тонкой ячейки (рис. 1). Заметим, что широкополосное излучение накачки может облучать данную ячейку в произвольном направлении.

Мы рассмотрели газовую ячейку в форме прямоугольного параллелепипеда, поскольку для такой геометрии существенно упрощаются расчеты. Однако полученные в настоящей работе качественные результаты справедливы также для тонких газовых ячеек другой формы (в частности, для цилиндрической ячейки с диаметром $d$ и внутренней толщиной $l \ll d)$. Сигнал поглощения от оптически возбужденных атомов может быть усилен при использовании серии последовательно расположенных тонких газовых ячеек. Необходимые для подобных экспериментов достаточно короткие световые импульсы (с длительностью вплоть до фемтосекунд) могут быть 
получены посредством широко используемых в спектроскопии лазеров [1].

\section{Конфликт интересов}

Автор заявляет, что у него нет конфликта интересов.

\section{Список литературы}

[1] Demtroder W. Laser Spectroscopy: Basic Concepts and Instrumentation. Springer-Verlag, 1996. 924 p.

[2] Izmailov A.Ch. // Az. J. Physics: Fizika. 2011. V. 17. N 1. Section: EN. P. 3. doi physics.gov.az/index1.html

[3] Mikata A., Tanaka U., Urabe S. // Appl. Opt. 2008. V. 47. N 5. P. 639.

[4] Izmailov A.Ch. // Las. Phys. 2009. V. 19. N 6. P. 1239.

[5] Измайлов А.Ч. // Опт. и спектр. 2018. Т. 124. В. 6. С. 732; Izmailov A.Ch. // Opt. Spectrosc. 2018. V. 124. N 6. P. 763.

[6] Radzig A.A., Smirnov B.M. Reference Data on Atoms, Molecules, and Ions. Berlin-Heidelberg: Springer-Verlag, 1985. $465 \mathrm{p}$

[7] Peyrot T., Sortais Y.R.P., Greffet J.-J., Browaeys A., Sargsyan A., Keaveney J., Hughes I.G., Adams C.S. // Phys. Rev. Lett. 2019. V. 122. N 11. P. 113401. 